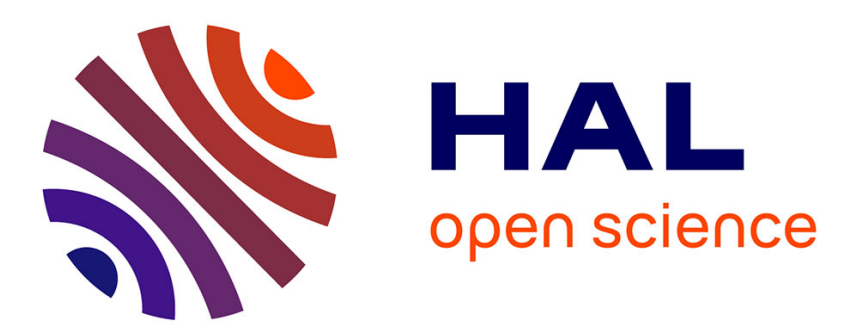

\title{
Fragilité en atmosphère d'hydrogène d'alliages à base de niobium
}

C. Vercaemer, R. Geschier, J. Guille, A. Clauss, H. Forestier

\section{To cite this version:}

C. Vercaemer, R. Geschier, J. Guille, A. Clauss, H. Forestier. Fragilité en atmosphère d'hydrogène d'alliages à base de niobium. Revue de Physique Appliquée, 1970, 5 (3), pp.437-442. 10.1051/rphysap:0197000503043700 . jpa-00243413

\section{HAL Id: jpa-00243413 https://hal.science/jpa-00243413}

Submitted on 1 Jan 1970

HAL is a multi-disciplinary open access archive for the deposit and dissemination of scientific research documents, whether they are published or not. The documents may come from teaching and research institutions in France or abroad, or from public or private research centers.
L'archive ouverte pluridisciplinaire HAL, est destinée au dépôt et à la diffusion de documents scientifiques de niveau recherche, publiés ou non, émanant des établissements d'enseignement et de recherche français ou étrangers, des laboratoires publics ou privés. 


\title{
FRAGILITÉ EN ATMOSPHÈRE D'HYDROGẼNE D'ALLIAGES A BASE DE NIOBIUM
}

\author{
par C. VERCAEMER, R. GESCHIER, J. GUILle, A. CLAUSS, et H. FORESTIER \\ Département Sciences de Matériaux, E. N. S. C. Strasbourg, \\ 1 , rue Blaise-Pascal, 67, Strasbourg
}

\begin{abstract}
Résumé. - Les alliages Nb-5 Mo, Nb-5 Ti, Nb-2 Zr, Nb-4 Zr et Nb-Zr, à l'état de grande pureté, manifestent à la température ambiante une certaine fragilité lorsqu'ils sont soumis à des essais de traction dans une atmosphère d'hydrogène. Il se dégage un parallélisme étroit entre l'influence des éléments alliants sur la susceptibilité du niobium à la fragilisation par l'hydrogène et l'influence de ces mêmes éléments sur le relèvement de la température de transition ductile-fragile. La contamination de ces alliages par l'oxygène ou l'azote accentue cette fragilité. Par contre, la présence de vapeur d'eau dans l'hydrogène peut la supprimer. Une tentative d'interprétation de l'ensemble des observations est donnée.
\end{abstract}

\begin{abstract}
The effect of hydrogen environment on embrittlement at room temperature of $\mathrm{Nb}$ rich, $\mathrm{Nb}-\mathrm{Mo}, \mathrm{Nb}-\mathrm{Zr}$ et $\mathrm{Nb}-\mathrm{Ti}$ alloys has been studied. We have observed a correlation between the effect of substitutional solutes on the raising of the ductile-to-brittle transition temperature and the effect of these substitutional solutes on the increasing of embrittlement in hydrogen environment. Interstitial solutes ( 0 and $N$ ) increase the embrittling effect of hydrogen and a profound effect of hydrogen gas purity on the phenomen has been noted. An explanation of the results is suggested.
\end{abstract}

Les solutions solides $\mathrm{Nb}-\mathrm{O}$ et $\mathrm{Nb}-\mathrm{N}$ peuvent présenter une grande fragilité lorsqu'elles subissent une déformation en atmosphère d'hydrogène à la température ambiante [1]. Parmi les différents paramètres susceptibles d'influencer les modalités d'apparition de cette fragilité, nous rappellerons les effets de la vitesse de déformation et de la concentration en atomes interstitiels : la capacité de déformation dans l'hydrogène, caractérisée par l'allongement à la rupture, est d'autant plus réduite que la vitesse de déformation est plus grande et que la teneur en interstitiels est plus élevée. Walter et Chandler constatent un phénomène analogue dans le cas de l'alliage de substitution B 66 qui comporte $5 \%$ de Mo, $5 \%$ de V et $1 \%$ de $\mathrm{Zr}$ [2]. Cette baisse de ductilité, préjudiciable à l'utilisation pratique du niobium, peut conduire également à des aspects théoriques intéressants.

Nous avons entrepris une étude plus générale de l'influence d'additions interstitielles et substitutionnelles sur le phénomène de fragilisation par l'hydrogène. Le travail rapporté ici s'insère dans le cadre d'une convention de recherche D. G. R. S. T. (Comité de Métallurgie) ayant pour objet l'étude de l'influence de certaines impuretés métalliques et non métalliques sur les propriétés des alliages de niobium.
Les alliages étudiés sont des solutions solides $\mathrm{Nb}-\mathrm{O}$ de différentes concentrations, cinq alliages binaires de substitution, et deux alliages ternaires $\mathrm{Nb}-\mathrm{Mo}-\mathrm{O}$ et $\mathrm{Nb}-\mathrm{Mo}-\mathrm{N}$ à teneurs variables en interstitiels. Après avoir indiqué les conditions expérimentales, nous décrirons le comportement mécanique, dans l'air et dans l'hydrogène, de ces différents alliages. L'ensemble des résultats expérimentaux nous conduira, enfin, à émettre quelques hypothèses sur le mécanisme de fragilisation et à comparer l'influence des éléments d'addition sur la fragilité dans l'hydrogène à leur incidence sur la température de transition ductilefragile.

I. Conditions expérimentales. - Afin de pouvoir déterminer l'influence spécifique des additions, il nous fallait disposer d'alliages à base de niobium dans un état de grande pureté. La contamination ultérieure, par l'oxygène ou l'azote, s'effectue de façon contrôlée. Les propriétés mécaniques sont déduites de l'essai de traction.

A. MatériauX De DéPART. - Les matériaux de départ sont le niobium pur et cinq alliages binaires de substitution $\mathrm{Nb}-5,3 \% \mathrm{Mo}, \mathrm{Nb}-4,65 \mathrm{Ti}, \mathrm{Nb}-2,0 \%$ $\mathrm{Zr}, \mathrm{Nb}-3,85 \% \mathrm{Zr}$ et $\mathrm{Nb}-5,45 \% \mathrm{Zr}$ (\% atomique). 
Ces alliages ont été préparés, par fusion, sous forme de lingots de très grande pureté. Leur transformation ultérieure en fil de $3 / 10 \mathrm{~mm}$ est effectuée intégralement à froid afin de limiter toute contamination. Les condi-

\begin{tabular}{|c|c|c|}
\hline & $\mathrm{O}_{\mathrm{ppm}}$ & $\mathrm{N}_{\mathrm{ppm}}$ \\
\hline - & - & - \\
\hline $\mathrm{Nb}-2 \mathrm{Zr}$ & 15 à 35 & 20 à 35 \\
\hline $\mathrm{Nb}-3,85 \mathrm{Zr} \ldots$ & 20 à 35 & 20 à 50 \\
\hline $\mathrm{Nb}-5,45 \mathrm{Zr} \ldots$ & 15 à 25 & 15 à 30 \\
\hline Nb-5, 3 Mo $\ldots \ldots \ldots$ & 25 à 45 & 40 à 60 \\
\hline $\mathrm{Nb}-4,65 \mathrm{Ti} \ldots \ldots \ldots$ & 70 à 110 & 50 à 80 \\
\hline
\end{tabular}

Les fils de 3/10 mm de diamètre, écrouis à 99, $95 \%$ après transformation, subissent un recuit de recristallisation, par effet Joule, sous un vide de $5 \times 10^{-7}$ torr. Les conditions de température et de durée de ce recuit sont adaptées à chaque alliage, afin de conférer à l'ensemble de nos matériaux une taille moyenne de grains correspondant à 1000 grains $/ \mathrm{mm}^{2}$.

B. Contamination CONTrolée en interstitiels. L'influence de la teneur en éléments interstitiels $(\mathrm{O}$ et $\mathrm{N}$ ) a été étudiée dans les cas du niobium et de l'alliage $\mathrm{Nb}$-Mo. L'oxygène et l'azote sont introduits en quantités contrôlées, par diffusion à haute température, après transformation des alliages en fil de $3 / 10 \mathrm{~mm}$. Cette technique de contamination présente deux avantages :

- la transformation du niobium et des alliages est beaucoup plus facile lorsqu'ils sont purs;

- les teneurs en oxygène et en azote peuvent être ajustées avec beaucoup de souplesse et choisies, tout au long de l'étude, dans un large domaine de concentration.

Les conditions de préparation et d'homogénéisation ont été précisées par ailleurs et sont complétées par une étude physico-chimique [4].

C. Conditions DE L'essai DE TRACtion. - Le comportement mécanique des échantillons est étudié à l'aide d'une micromachine de traction SADAMEL Mi 44/2. Lorsque l'essai de traction est effectué dans l'hydrogène, l'éprouvette se trouve dans une chambre dans laquelle règne une pression d'hydrogène de l'atmosphère. Les essais sont effectués à température ambiante.

Des études antérieures ayant mis en évidence une augmentation de la fragilité en fonction de la vitesse de déformation, nous avons effectué la totalité des essais à la vitesse de traction maximum de la machine. Compte tenu de la longueur utile des éprouvettes, leur vitesse de déformation réelle est de $1,2 \times 10^{-1} \mathrm{~s}^{-1}$.

Par ailleurs, certains auteurs ont mis en évidence l'importance très grande de la pureté de l'hydrogène sur la perméabilité du niobium et de ses alliages vis-àvis de ce gaz [5]. Tenant compte de cette observation, nous avons utilisé de l'hydrogène de qualité « $U$ » tions précises d'élaboration et de transformation de ces alliages font l'objet d'un autre mémoire [3].

Les résultats des analyses effectuées sur les différents alliages sont résumés dans le tableau ci-après :

$\begin{array}{ccc}\mathrm{C}_{\mathrm{ppm}} & \mathrm{H}_{\mathrm{ppm}} & \begin{array}{c}\sum \text { des impure } \\ \text { métalliques p }\end{array} \\ 15 \text { à } 25 & - & - \\ 15 \text { à } 25 & 2 & <200 \\ 10 \text { à } 20 & 2 & <200 \\ 15 \text { à } 20 & 2 & <200 \\ 25 \text { à } 35 & 2 & <200 \\ & & <200\end{array}$

et avons toujours purgé longuement la chambre d'essai avant de procéder à l'essai de traction.

La principale caractéristique mécanique à laquelle nous nous référerons est l'allongement à la rupture.

Nous caractériserons la fragilité dans l'hydrogène

- soit en comparant les valeurs des allongements à la rupture des échantillons rompus dans l'air et dans l'hydrogène,

- soit en considérant un taux de fragilité défini par :

$$
\frac{A \% \text { oair }-A \% \text { hydrogène }}{A \% \text { air }} \times 100 .
$$

II. Comparaison du comportement mécanique, dans l'air et dans l'hydrogène, des différents alliages. Nous avons envisagé successivement l'influence de l'addition d'un élément en insertion $(\mathrm{O})$, de trois éléments en substitution ( $\mathrm{Ti}, \mathrm{Zr}$ et $\mathrm{Mo}$ ) et de l'addition simultanée d'un élément en insertion ( $\mathrm{O}$ ou $\mathrm{N}$ ) et d'un élément en substitution (Mo) sur les propriétés mécaniques du niobium dans l'air et dans l'hydrogène. Les principaux résultats de cette étude expérimentale sont rassemblés ci-après.

A. Alliages Nb-O. - La fragilité des solutions solides $\mathrm{Nb}-\mathrm{O}$ en atmosphère d'hydrogène a déjà été étudiée par R. Kieger [1]. Cependant, le degré de pureté du métal utilisé par cet auteur était médiocre et l'atmosphère d'hydrogène insuffisamment définie. Aussi avons-nous décidé de refaire cette étude dans les conditions expérimentales précédemment définies et de comparer nos résultats à ceux obtenus antérieurerement.

Sur la figure 1 nous avons reporté les valeurs de l'allongement à la rupture, dans l'air et dans l'hydrogène, en fonction de la teneur en oxygène. Les deux séries d'expériences conduisent aux mêmes conclusions qualitatives et mettent en évidence l'existence d'un seuil de concentration en oxygène en dessous duquel l'effet de l'hydrogène ne se manifeste pas. Cependant, ce seuil est très différent d'une étude à l'autre.

Les deux séries de solutions solides étudiées présentent donc une susceptibilité à la fragilisation par 


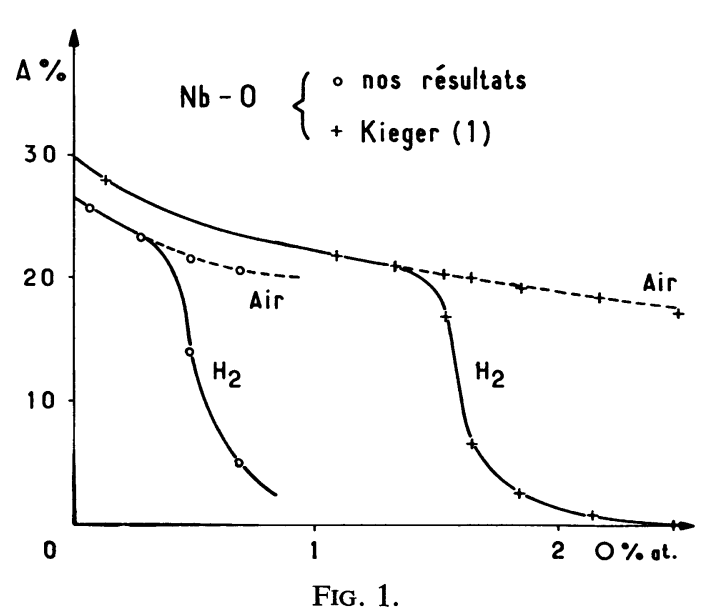

l'hydrogène nettement différente. Cette constatation souligne la nécessité impérieuse de caractériser le plus complètement possible le matériau du point de vue physico-chimique et structural, de préciser les conditions de l'essai et de définir la nature de l'environnement.

Nous pensons que l'explication réside dans la considération des deux facteurs suivants :

- D'une part la taille des grains diffère notablement pour les deux séries d'expérience : 6400 grains/ $\mathrm{mm}^{2}$ et 1000 grains $/ \mathrm{mm}^{2}$. Or, Kieger a montré que la tendance à la fragilité augmente lorsque la taille des grains augmente.

Notons que la grande pureté de notre niobium entraîne une recristallisation importante à des températures inférieures à la température d'introduction de l'oxygène. De ce fait nous n'avons pas pu préparer des solutions solides $\mathrm{Nb}-\mathrm{O}$ ayant une taille de grains voisine de celle des échantillons de Kieger. Ce facteur métallurgique explique également les différences observées en ce qui concerne l'allongement dans l'air: nos échantillons ayant une taille de grains plus grande ont un allongement dans l'air plus faible.

- D'autre part, l'hydrogène que nous avons utilisé est très pur. Nous montrerons en effet, ultérieurement, que des traces de vapeur d'eau dans l'hydrogène peuvent déplacer considérablement le seuil de concentration correspondant à l'apparition de la fragilité.

B. Alliages de substitution. - Des essais de traction, dans l'air et dans l'hydrogène, ont été effectués sur les cinq alliages de substitution. Leur allongement à la rupture dans l'air est comparable et se situe entre 20 et $25 \%$. Tous ces alliages présentent, à l'état pur, une fragilité marquée dans l'hydrogène.

La figure 2 résume l'ensemble des résultats obtenus : le "taux de fragilité », qui est représenté en fonction de la limite élastique de l'alliage, caractérise nettement l'effet de l'hydrogène. A titre de comparaison nous avons également reporté les valeurs de l'alliage $\mathrm{Nb}-\mathrm{O}$. L'examen de cette figure conduit aux remarques suivantes :

- Lorsque la concentration en élément substitutionnel augmente, le taux de fragilité augmente (cas

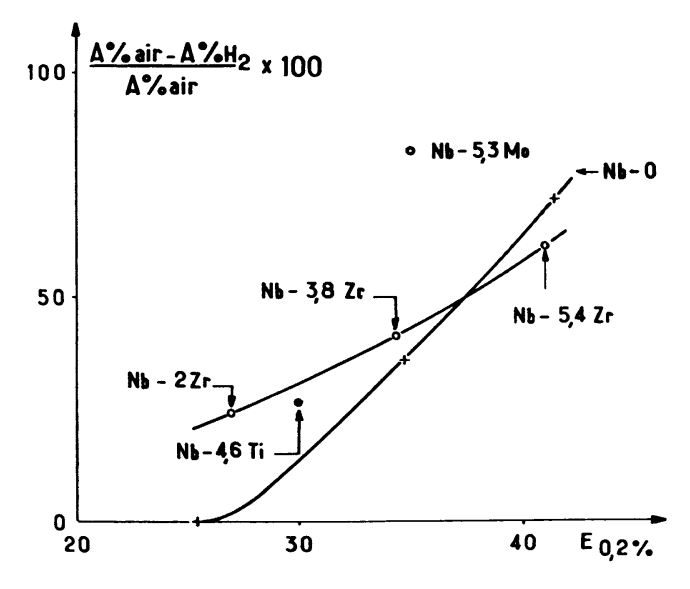

FIG. 2.

des alliages $\mathrm{Nb}-\mathrm{Zr}$ ). Il en est d'ailleurs de même lorsque l'addition se fait par insertion (alliages $\mathrm{Nb}-\mathrm{O}$ ). Le taux de fragilité croît régulièrement, dans les deux cas, en fonction de la limite élastique.

- A teneurs en éléments de substitution approximativement égales ( $\mathrm{Nb}-4,6 \mathrm{Ti}, \mathrm{Nb}-5,4 \mathrm{Zr}, \mathrm{Nb}-5,3 \mathrm{Mo}$ ), le taux de fragilité est fonction de la nature de l'addition et croît du titane au zirconium et au molybdène. La variation du taux de fragilité n'est plus une fonction monotone de la limite élastique : des trois éléments de substitution envisagés, c'est le molybdène qui a l'action fragilisante la plus marquée.

Il résulte de ces observations que des éléments de substitution peuvent jouer un rôle comparable aux éléments d'insertion dans le processus de fragilisation par l'hydrogène. Il apparaît également que la limite élastique n'est pas le seul facteur déterminant ; d'autres paramètres, tels la concentration électronique par exemple, peuvent jouer un rôle prépondérant dans le phénomène étudié.

C. AdDition Simultanée D'Un ÉlÉMent InTERSTITIEL ET D'Un ÉLÉMENT DE SUBSTITUTION. - Cette étude a été faite sur l'alliage $\mathrm{Nb}-5,3 \mathrm{Mo}$ auquel on a ajouté des quantités contrôlées d'oxygène ou d'azote. Les essais de traction ont été effectués dans l'air, dans l'hydrogène pur et dans l'hydrogène humide. Dans ce dernier cas, l'hydrogène traverse un flacon laveur rempli d'eau avant de parvenir dans la chambre d'essai. La figure 3 relative aux alliages $\mathrm{Nb}-\mathrm{Mo}-\mathrm{O}$ et la figure 4 relative aux alliages $\mathrm{Nb}-\mathrm{Mo}-\mathrm{N}$ résument les résultats. Les conclusions, basées sur la considération de l'allongement à la rupture, sont très analogues pour les deux figures :

- Lorsque les essais sont effectués dans l'hydrogène $U$ pur, l'addition d'un interstitiel accentue encore, quoique dans une faible mesure, la fragilité de l'alliage.

- La présence de vapeur d'eau dans l'hydrogène diffère considérablement l'apparition de la fragilité dans le cas des alliages $\mathrm{Nb}-\mathrm{Mo}-\mathrm{O}$ et la supprime totalement dans le cas des alliages Nb-Mo-N. 


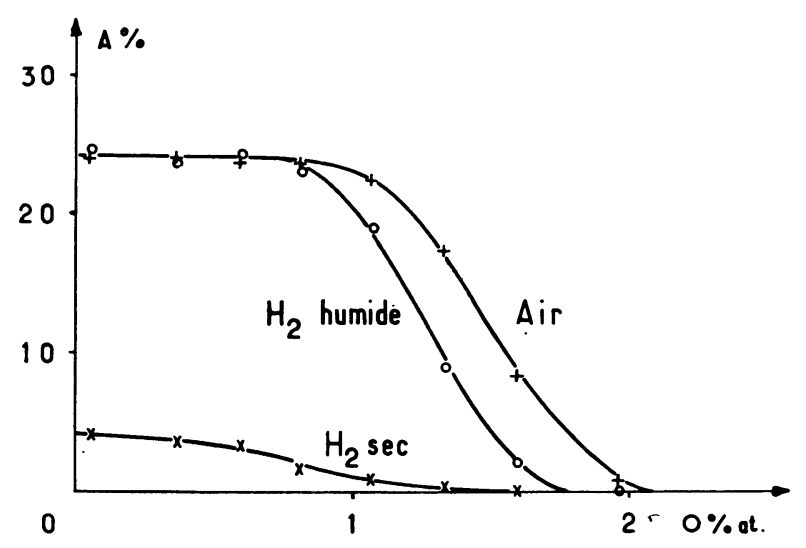

FIG. 3.

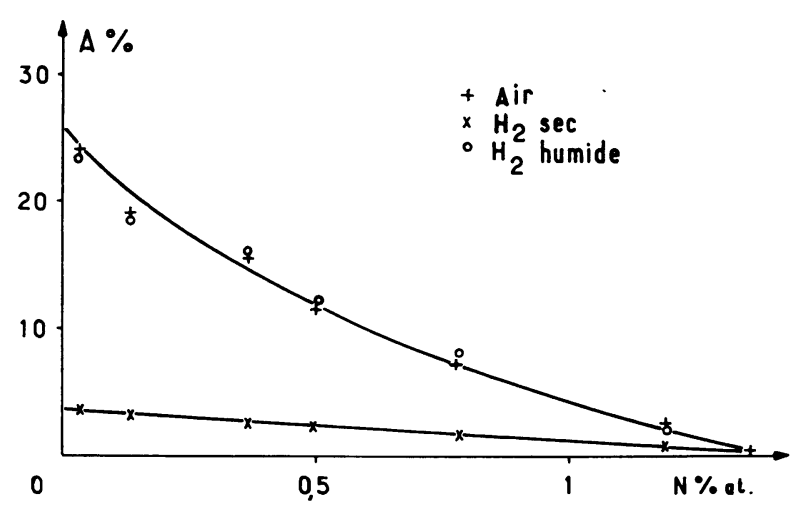

FIG. 4.

Cette dernière remarque permet d'envisager l'influence possible d'autres impuretés contenues dans l'hydrogène et pourrait expliquer, du moins dans une certaine mesure, les différences de fragilité observées dans le cas des solutions solides $\mathrm{Nb}-\mathrm{O}$ considérées au début de cette étude.

L'ensemble de ces résultats généralise les observations antérieures en établissant clairement l'existence d'une fragilité quel que soit l'élément alliant, qu'il soit en insertion $(\mathrm{O}, \mathrm{N})$ ou en substitution $(\mathrm{Mo}, \mathrm{Zr}$, Ti), qu'il augmente la concentration électronique de l'alliage $(\mathrm{Mo}, \mathrm{O}, \mathrm{N})$ ou qu'il la diminue $(\mathrm{Ti}, \mathrm{Zr})$, qu'il dilate la maille du réseau $(\mathrm{O}, \mathrm{N}, \mathrm{Zr})$ ou qu'il la contracte $(\mathrm{Mo})$. Cependant, le Niobium pur ne présente pas de fragilité dans les conditions d'essai; une teneur minimum en élément alliant paraît donc nécessaire.

III. Tentative d'interprétation. - Avant d'envisager un mécanisme possible de la fragilisation en atmosphère d'hydrogène, il nous paraît intéressant de rendre compte de quelques observations complémentaires permettant de mieux situer le phénomène.

A. Remarques préliminaires. - L'examen microfractographique des surfaces de rupture montre clairement la modification du type de rupture lorsque la fragilité apparaît : dans l'air, les surfaces de rupture révèlent de nombreuses cupules correspondant à une déformation importante; dans l'hydrogène, le faciès est celui d'une rupture fragile, par clivage. L'exament micrographique d'une section perpendiculaire à la surface de rupture confirme le caractère intragranulaire de la rupture.

Par ailleurs, la fragilité en atmosphère d'hydrogène augmente en fonction de la vitesse de déformation. En fait, l'augmentation de la vitesse de déformation relève la limite élastique et Kieger avait émis l'hypothèse que, à diamètre de grain donné, le durcissement du matériau, quelle qu'en soit l'origine, était le critère le plus important de l'apparition de la fragilité en atmosphère d'hydrogène. Nos résultats nous conduisent à nuancer cette hypothèse : les différentes additions faites au niobium ont pour effet commun de durcir le métal, mais il apparaît que la limite élastique n'est pas le seul paramètre déterminant le taux de fragilité. D'autres facteurs interviennent, tels par exemple la concentration électronique de l'alliage dont dépendent l'énergie de cohésion du métal [6] et, très certainement, l'énergie d'interaction métal-hydrogène.

Enfin, la fragilité des alliages de niobium étudiés ne se manifeste que si l'hydrogène est présent au moment même de la déformation et jusqu'à son stade ultime, la rupture. Ainsi, une éprouvette abandonnée longuement en atmosphère d'hydrogène pur, puis tractionnée à l'air, ne présente aucune fragilité.

Cette constatation, ainsi que l'influence de la vapeur d'eau présente dans l'hydrogène, sont à rapprocher d'autres observations faites lors d'études sur l'adsorption et la perméabilité à l'hydrogène des métaux réfractaires. Ces études mettent l'accent sur l'importance de la pureté du gaz et de l'état de surface, particulièrement aux températures inférieures à $500^{\circ} \mathrm{C}$ et pour des pressions d'hydrogène de l'ordre de une atmosphère [5]. La réactivité vis-à-vis de l'hydrogène d'une surface, activée par recuit sous vide à haute température, peut devenir supérieure d'un facteur $10^{4}$ à celle d'une surface brute; l'activation résulterait de l'enlèvement de la fine pellicule d'oxyde superficielle.

$\mathrm{Au}$ cours d'un essai de traction, seules les surfaces neuves, qui apparaissent au cours de la déformation, seraient par conséquent suffisamment réactives vis-àvis de l'hydrogène. Ceci explique la nécessité de la présence de l'hydrogène au moment de la traction et l'influence d'impuretés telles $\mathrm{H}_{2} \mathrm{O}$ (et vraisemblablement l'oxygène et d'autres impuretés) présentes dans le gaz et susceptibles d'entrer en compétition avec l'hydrogène pour réagir avec les surfaces neuves créées.

B. INTERPRÉTATION DES PRINCIPAUX FAITS OBSERVÉS. Le mécanisme de la rupture fragile par clivage peut être décomposé en 3 phases :

- la germination de microfissures par coalescence de dislocations ;

- la croissance de ces microfissures jusqu’à ce 
que l'une d'entre elles atteigne une dimension satisfaisant à la condition de Griffith et Orowan

$$
\sigma=\left(\frac{2 E p}{\pi C}\right)^{1 / 2}
$$

$C=$ longueur de la fissure.

$P=$ somme de l'énergie de surface de la fissure et de l'énergie consommée par déformation plastique lors de sa propagation.

- L'extension de cette fissure jusqu'à la rupture.

De ces trois phases, il semble que la seconde soit déterminante. Dans le cas de la rupture ductile, telle qu'elle se manifeste lors des tractions effectuées à l'air, les microfissures formées se résorbent par écoulement plastique avant d'atteindre la dimension critique. Quelle est l'influence possible d'une atmosphère d'hydrogène sur le mécanisme décrit ci-dessus ?

La germination d'une microfissure par coalescence de dislocations est un processus qui se déroule au sein d'un grain. Envisager l'intervention de l'hydrogène sur la phase initiale de propagation de la microfissure revient, par conséquent, à admettre une diffusion de l'hydrogène dans le réseau du métal dans les conditions de l'essai de traction. Une telle diffusion, rapide et limitée à une profondeur de quelques dizaines de couches atomiques, est concevable dans le cas d'un réseau très perturbé proche de la surface de l'éprouvette. En effet, le coefficient de diffusion de l'hydrogène est très sensible à l'état de contrainte statique, et à fortiori dynamique, du réseau.

L'hydrogène présent dans le métal intervient soit à l'état dissous (théorie de Troiano), soit à l'état adsorbé sur les lèvres des microfissures, l'abaissement de l'énergie de surface diminuant la tension nécessaire à leur propagation. Son effet peut être comparé à l'effet observé lors d'études sur la température de transition ductile-fragile : l'hydrogène présent dans le niobium, même à faible concentration, confère au métal une tendance accrue au clivage et relève considérablement la température de transition [7].

Que sa propagation soit favorisée ou non par la diffusion préalable d'hydrogène, lorsqu'une microfissure, formée à proximité immédiate de la surface, débouche sur celle-ci, l'hydrogène présent dans l'atmosphère s'adsorbe fortement sur ses parois métalliques fraîches et favorise sa propagation en diminuant l'énergie de surface.

Parmi les différents facteurs métallurgiques susceptibles de jouer un rôle, on peut affirmer que, d'une façon générale, tout ce qui perturbe l'écoulement plastique, donc tout ce qui favorise la formation même très localisée de contraintes élastiques élevées, favorise la fragilisation par l'hydrogène atmosphérique. En effet, l'existence de contraintes élevées est favorable à la diffusion de l'hydrogène; elle l'est également à l'extension de la microfissure aux dépens de la déformation plastique qui nécessite des tensions plus élevées. Ces observations permettent en particulier d'expliquer les influences de la vitesse de déformation et de la concentration en interstitiels sur la susceptibilité du niobium à la fragilisation par l'hydrogène gazeux.

Le rôle des éléments alliants de substitution est moins clair. Dans ce cas, comme nous l'avions souligné, le durcissement ne permet pas à lui seul d'expliquer l'ensemble des résultats. Cependant, la comparaison de nos résultats avec ceux obtenus par d'autres chercheurs qui ont étudié l'influence de différentes additions substitutionnelles au niobium et au tantale sur la température de transition ductile-fragile [8], permet de mettre en évidence une analogie frappante : l'action des additions croît dans le même ordre, du titane au zirconium et au molybdène dans les deux types d'études.

Il résulte de cet ensemble de considérations que les différents facteurs qui relèvent la température de transition ductile-fragile favorisent de façon très analogue la fragilité en atmosphère d'hydrogène. Une telle analogie permet de prévoirl a susceptibilité à la fragilisation par l'hydrogène des différents alliages de niobium dès lors que l'on peut comparer leur température de transition ductile-fragile.

Les résultats de la présente étude permettent de généraliser aux alliages de niobium de substitution le phénomène de fragilisation par l'hydrogène observé antérieurement dans le cas de solutions solides d'insertion. En considérant l'influence d'un certain nombre de paramètres sur les modalités d'apparition de cette fragilité, on a pu mettre en évidence un parallélisme très étroit entre ce phénomène et la variation de la température de transition ductile-fragile de ces mêmes matériaux. Enfin, les impuretés gazeuses présentes dans l'hydrogène peuvent jouer un rôle bénéfique et conduire à la suppression totale de tout effet fragilisant dû à l'hydrogène.

\section{Bibliographie}

[1] KIEger (R.), Thèse Sciences, Strasbourg, 1968.

Kieger (R.), Clauss (A.) et Forestier (H.), Mém. Sci. Rev. Métallurg., 1967, 64, 195.

Clauss (A.), Kieger (R.), Schmuck (J.), Colloque sur l'hydrogène dans les métaux, VALDUC, 1967.
[2] Walter (R. J.) et Chandler (W. T.), AIAA J., 1966, 4, 302.

[3] Geschier (R.), Givord (J. P.), Armand (M.), Clauss (A.) et Forestier (H.), Journées du Niobium, 1969. 
[4] Vercaemer (C.) et Clauss (A.), C. R. Acad. Sci., $1969,269,803$.

Vercaemer (C.), Geschier (R.), Guille (J.) et Clauss (A.), C. R. Acad. Sci., à paraître.

Geschier (R.), Vercaemer (C.), Guille (J.) et Clauss (A.), C. R. Acad. Sci., à paraître.

[5] Albrecht (W. M.), Goode (W. D.) et Mallett (M. W.), J. Electrochem. Soc., 1959, 106, 981.

Gulbransen (E. A.) et ANDREW (K. F.), J.Electrochem. Soc., 1954, 101, 348.
[6] Blanchard (P.) et Troiano (A. R.), Mém. Sci. Rev. Métallurg., 1960, 57, 409.

[7] Eustice (A. L.), et Carlson (O. H.), Trans. ASME, 1961, 53, 501.

Wilcox (B. A.), Brisbane (A. W.) et Klinger (R. F.), Trans. ASME, 1962, 55, 179.

[8] Begley (R. T.) et Bechtold (J. H.), J. Les Commons Metals, 1961, 3, 1. 